\title{
The Increased Level of Depression and Anxiety in Irritable Bowel Syndrome Patients Compared with Healthy Controls: Systematic Review and Meta-analysis
}

\begin{abstract}
Changhyun Lee, ${ }^{1}$ Eunyoung Doo, ${ }^{1}$ Ji Min Choi, ${ }^{1}$ Seung-ho Jang, ${ }^{2}$ Han-Seung Ryu, ${ }^{3}$ Ju Yup Lee, ${ }^{4}$ Jung Hwan Oh, ${ }^{5}$ Jung Ho Park, ${ }^{6}$ and Yong Sung Kim ${ }^{7 *}$; Brain-Gut Axis Research Group of Korean Society of Neurogastroenterology and Motility

${ }^{1}$ Department of Internal Medicine and Healthcare Research Institute, Healthcare System Gangnam Center, Seoul National University Hospital, Seoul, Korea; ${ }^{2}$ Department of Psychiatry, School of Medicine, Wonkwang University, Iksan, Jeollabuk-do, Korea; ${ }^{3}$ Department of Gastroenterology and Digestive Disease Research Institute, Wonkwang University School of Medicine, Iksan, Jeollabuk-do, Korea; ${ }^{4}$ Department of Internal Medicine, Keimyung University School of Medicine, Daegu, Korea; ${ }^{5}$ Division of Gastroenterology, Internal Medicine, College of Medicine, The Catholic University of Korea, Seoul, Korea; ${ }^{6}$ Department of Medicine, Samsung Medical Center, Sungkyunkwan University School of Medicine, Seoul, Korea; and ${ }^{7}$ Division of Gastroenterology, Department of Internal Medicine, Wonkwang University Sanbon Hospital, Gunpo, Gyeonggi-do, Korea
\end{abstract}

\section{Background/Aims}

Irritable bowel syndrome (IBS) patients commonly experience psychiatric disorders, such as depression and anxiety. This meta-analysis sought to compare depression and anxiety levels between IBS patients and healthy controls.

\section{Methods}

We searched major electronic databases (PubMed, EMBASE, MEDLINE, and Cochrane library) to find comparative studies on IBS patients and healthy controls. The primary outcome was a standardized mean difference (SMD) of anxiety and depression levels; subgroup analyses were conducted according to IBS-subtypes.

\section{Results}

In total, 2293 IBS patients and 4951 healthy controls from 27 studies were included. In random effect analysis, depression and anxiety levels were significantly higher in IBS patients (pooled SMD $=0.76 ; 95 \% \mathrm{Cl}, 0.62-0.90 ; P<0.001 ; P^{2}=77.2 \%$ and pooled SMD = $0.84 ; 95 \% \mathrm{Cl}, 0.67-1.01 ; P<0.001 ; I^{2}=85.6 \%$, respectively). Both analyses' funnel plots showed symmetry. In meta-regression analysis, heterogeneity was due to the studied region and questionnaire type for both depression and anxiety. In sub-group analyses of IBS-subtype, the pooled SMDs of depression and anxiety levels (IBS with predominant constipation: 0.83 and 0.81 , IBS with predominant diarrhea: 0.73 and 0.65 , and IBS with mixed bowel habits: 0.62 and $0.75 ; P<0.001$, respectively) were significantly higher in all IBS-subtypes.

\section{Conclusions}

The present meta-analysis showed depression and anxiety levels to be higher in IBS patients than in healthy controls, regardless of IBSsubtype. However, the gender effect on psychological factors among IBS patients could not be determined and should be evaluated in prospective studies.

(J Neurogastroenterol Motil 2017;23:349-362)

Key Words

Anxiety; Depression; Irritable bowel syndrome; Meta-analysis

Received: December 26, 2016 Revised: June 8, 2017 Accepted: June 10, 2017

(a) This is an Open Access article distributed under the terms of the Creative Commons Attribution Non-Commercial License (http://creativecommons. org/licenses/by-nc/4.0) which permits unrestricted non-commercial use, distribution, and reproduction in any medium, provided the original work is properly cited.

${ }^{*}$ Correspondence: Yong Sung Kim, MD, PhD

Division of Gastroenterology, Department of Internal Medicine, Wonkwang University Sanbon Hospital, 327 Sanbon-ro, Gunpo, Gyeonggi-do 15865, Korea

Tel: +82-31-390-2973, Fax: +82-31-390-2244, E-mail: wms89@hanmail.net

Changhyun Lee and Eunyoung Doo contributed equally to this article 


\section{Introduction}

The Rome IV criteria defined functional gastrointestinal (GI) disorders (FGID) as "disorders of gut-brain interaction" because symptoms are generated not only in the gut, but also by the complex interactions among factors such as gut dysbiosis, altered gut signaling, and dysregulation of the central nervous system. ${ }^{1}$ Irritable bowel syndrome (IBS) is the most prevalent FGID, affecting approximately $10-15 \%$ of populations; diagnosis requires recurrent abdominal pain associated with $\geq 2$ of the following: (1) defecation, (2) a change in stool frequency, and (3) a change in stool form. ${ }^{2,3}$

Psychological problems such as anxiety and depression are common in IBS patients. ${ }^{4}$ Overlap between depression and FGID is about $30 \%$ and anxiety disorders are the most common psychiatric comorbidity in FGID patients. ${ }^{4,5}$ The psychosocial modifiers of IBS were emphasized in the "multi-dimensional clinical profile" introduced by the Rome IV criteria $^{1}$ because psychological factors influence IBS symptoms, modify illness behavior (eg, healthcare seeking), and contribute to poor outcomes. ${ }^{1}$

The brain-gut axis is the hard-wiring between the brain and gut, communicating information from the brain's emotional network (eg, from the cingulate cortex to the peripheral GI tract and vice versa). Therefore, the perception of pain is influenced by the emotional mechanism; IBS symptoms can also be affected by the psychological state. ${ }^{6,7}$ Treatment directed towards psychiatric conditions can reduce IBS symptoms' severity. ${ }^{8}$ Furthermore, psychosocial factors such as the psychological state, individual's traits, and life stress also affect the gut physiology through the brain-gut axis in the biopsychosocial model of IBS. ${ }^{4}$ Psychosocial factors impair mucosal secretory and barrier function through alteration of the efferent autonomic nervous system and the stress hormone system (hypothalamo-pituitary-adrenal axis), resulting in the translocation of bacterial cell products. ${ }^{1,4,9}$ Animal studies have demonstrated the influence of stress on colonic permeability, and mucosal and systemic inflammation's mediation by the autonomic nervous system and the hypothalamo-pituitary-adrenal axis. ${ }^{4}$

Several recently published studies have mapped IBS's relationship with psychological disorders ${ }^{10-16}$ and compared depression and anxiety levels in IBS-subtypes. ${ }^{15,17,18}$ However, the relationship between IBS and psychological disorders (depression or anxiety) was inconsistent, even in a recent meta-analysis entailing eight studies. ${ }^{19}$ The heterogeneity of the results was too high, with no further analysis of its cause. Furthermore, IBS-subtypes failed to show statistical significance for both anxiety and depression, except IBS with predominant constipation (IBS-C) and IBS with predominant diarrhea (IBS-D) for anxiety, and IBS-D for depression, because few studies were included.

Knowledge of IBS's correlation with psychiatric disorders would enhance understanding and treatment of IBS patients, as psychological distress could exacerbate symptoms, negatively affect treatment outcomes, and affect doctor-patient relationships. ${ }^{4}$ The aim of this meta-analysis was to compare depression and anxiety levels between IBS patients and healthy controls, also considering IBS subtypes and patient gender.

\section{Materials and Methods}

The protocol for this review was prospectively developed, detailing specific patients, healthy controls, the primary outcome, study selection criteria, and study quality checks. The checklist and flow chart of the Preferred Reporting Items for Systematic Review and Meta-Analyses (PRISMA) statement were referred to, to achieve the highest standard in reporting items for a systematic review and meta-analysis.

\section{Searching Strategy}

The following inclusion criteria were applied: (1) articles published from January 2000 to July 2016 in peer-reviewed journals, (2) comparative studies on IBS patients and healthy controls, (3) adults $\geq 18$ years, and (4) depression and anxiety levels presented or extracted as mean $\pm \mathrm{SD}$. Our search terms were "((irritable bowel syndrome) OR irritable bowel syndrome[MeSH Terms]) AND (((depression) OR depression [MeSH Terms]) OR depressive disorders [MeSH Terms])) AND (((anxiety) OR anxiety disorders[MeSH Terms]) OR anxiety[MeSH Terms]))) AND “2000/01/01”[Date - Publication] : “2016/07/31”[Date - Publication]” for PubMed; “'irritable bowel syndrome' AND ('depressive disorder' OR 'depression') AND ('anxiety disorder' OR 'anxiety') AND 'article'/it AND [1-1-2000]/sd NOT [31-7-2016]/ sd AND [embase]/lim” for Embase; “irritable bowel syndrome' AND ('depressive disorder' OR 'depression') AND ('anxiety disorder' OR 'anxiety') AND [2000-2016]/py NOT [31-7-2016]/ sd AND 'article'/it AND [medline]/lim” for Medline; “"irritable bowel syndrome" in Title, Abstract, Keywords and "anxiety disorder" OR "anxiety" in Title, Abstract, Keywords and "depression" OR “depressive disorder" in Title, Abstract, Keywords" in Cochrane Reviews for Cochrane library. 


\section{Study Selection}

The results from the database search were imported into EndNote X7 software (Thomson Reuters, Philadelphia, PA, USA) and combined to remove duplicates. Two authors (C.L. and E.D.) independently assessed the titles and abstracts of all the studies identified through the search outlined above. Full texts of studies fulfilling the inclusion criteria were obtained and reviewed. Exclusion criteria were (1) studies did not present depression or anxiety scores, (2) studies that could not determine the mean and SD, (3) studies with no healthy controls, (4) studies with only one IBSsubtype or only post-infectious IBS, (5) studies with low quality scores, and (6) duplicated study data. Disagreements were resolved through discussion; otherwise the co-author, Y.S.K., was consulted.

\section{Assessing Methodological Quality}

Two independent authors (C.L. and E.D.) assessed article quality according to the Newcastle-Ottawa Scale for "case-control studies." ${ }^{20}$ The quality scale ranges from 0 to 9 points, with $\leq 4$ indicating poor article quality, and warranting its exclusion.

\section{Data Extraction}

Two authors (C.L. and E.D.) independently analyzed articles and extracted data using a pre-defined data extraction worksheet. The following information was extracted from each article: title, first author, publication year, country of origin, IBS criteria, type of questionnaire for depression, type of questionnaire for anxiety, the number of IBS patients, the number of healthy controls, the proportion of females in the IBS group, the mean and SD of depression levels, and the mean and SD of anxiety levels. To determine depression and anxiety levels in IBS-subtypes, the following information was also extracted: the number of IBS-subtype patients and means and SDs of depression and anxiety levels among these. In this meta-analysis, the standardized mean difference (SMD) was used for comparing IBS patients' and healthy controls' anxiety and depression levels because various types of questionnaires were used to assess these variables. We estimated SD from the standard error (SE) of the mean, using the formula $\mathrm{SD}=\mathrm{SE} \times \sqrt{\mathrm{N}}$, and calculated SD from a $95 \%$ confidence interval (CI) using the formula $\mathrm{SD}=\sqrt{\mathrm{N}} \times($ Upper limits - Lower limits $) \div 3.92$.

\section{Statistical Analysis and Risk of Bias Assessment}

Meta-analysis was conducted using STATA ver. 14.1 (Stata Corp, College Station, TX, USA). We used the STATA "metan" command for calculating SMD with $95 \%$ confidence intervals. If the studies showed substantial heterogeneity $\left(I^{2}>50 \%\right)$, we used random effects models. We also used the STATA “metan” command to create the forest plot. Publication bias was assessed through the Egger's test, using the "metabias" command, and presented graphically with funnel plots, using the "metafunnel" command. The cause of heterogeneity was analyzed using the "metareg" command.

\section{Results}

\section{Search Results}

Through a database search, 1737 records were identified (PubMed, $\mathrm{n}=637$; Embase, $\mathrm{n}=$ 579; Medline library, $\mathrm{n}=$ 517; Cochrane library, $\mathrm{n}=1$; other sources, ${ }^{21-23} \mathrm{n}=3$ ) and 837 records remained after duplicates were removed. Initial screening of the title and abstracts resulted in 786 records being excluded. Full texts of the remaining 51 records were reviewed in detail. A total of 27 articles were included in this systemic review and metaanalysis, ${ }^{10-13,15-17,21-40}$ and 24 articles were excluded with specific reasons; (1) 8 studies without depression or anxiety scores, ${ }^{41-48}$ (2) 3 studies from which the mean and SD could not be determined, ${ }^{49-51}$ (3) 4 studies with no healthy controls, ${ }^{14,52-55}$ (4) 2 studies with only one IBS-subtype or post-infectious IBS, ${ }^{56,57}$ (5) 5 studies with low quality scores (Newcastle-Ottawa Scale $\leq 4),{ }^{58-62}$ and (6) 2 studies with duplicated data. ${ }^{63,64}$ The selection process according to the PRISMA flow diagram is shown in Figure 1.

\section{Baseline Characteristics of Included Studies}

A total of 2293 IBS patients and 4951 healthy controls were included from 27 studies. ${ }^{10-13,15-17,21-40}$ The baseline characteristics of included studies are shown in Table 1 and the major findings were listed in Supplementary Table. Eight studies were conducted in East Asia, ${ }^{11-13,16,26,31,37,40} 7$ in the USA, ${ }^{10,17,21-23,30,33} 7$ in Europe, ${ }^{15,27,29,32,34-36}$ and 5 in the other regions. ${ }^{24,25,28,38,39}$ Three studies were cross-sectional, ${ }^{22,24,31} 11$ were age-sex matched casecontrol studies, ${ }^{11,12,15,16,21,25-27,37,40} 9$ were unmatched case-control studies, ${ }^{10,13,23,28,29,34,35,38,39}$ and 4 were case-control studies that enrolled female participants only. ${ }^{17,30,32,33}$ IBS was diagnosed using the Rome III criteria in 10 studies, ${ }^{10-13,15,16,32,35,39,40}$ Rome II criteria in 11 studies, ${ }^{17,21,24,28,29,31,33,34,36-38}$ Bowel Disease Questionnaire in 3 studies, ${ }^{22,23,30}$ Manning's criteria in 2 studies, ${ }^{25,27}$ and the Rome I criteria in 1 study. $^{26}$ The level of depression was assessed using the Hospitalization Anxiety and Depression Scale (HADS) in 14 studies, ${ }^{10,15-17,21,25,27,28,32,34,37-40}$ Beck Depression Inventory (BDI) in 


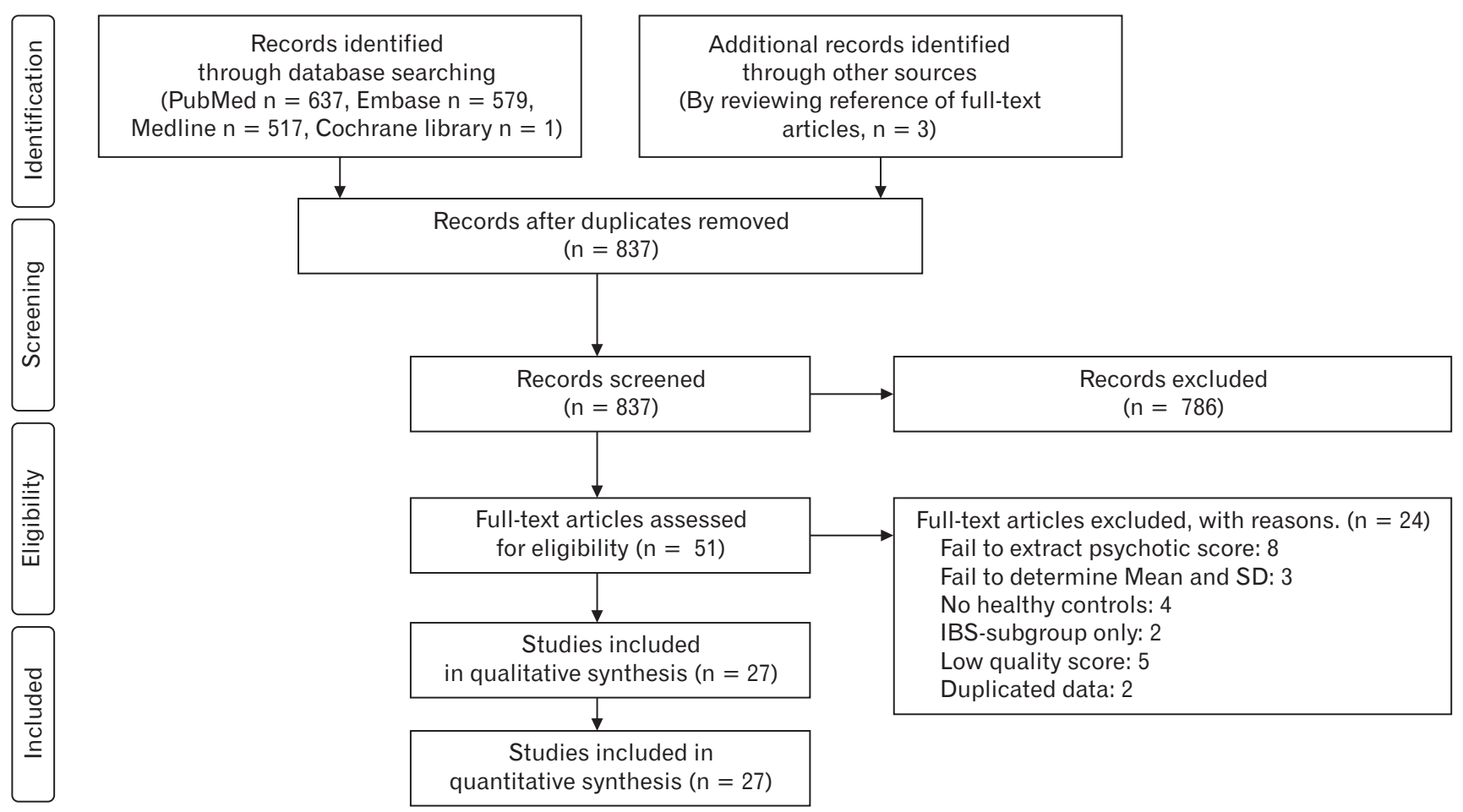

Figure 1. PRISMA flow diagram of the meta-analysis. IBS, irritable bowel syndrome.

5 studies, ${ }^{26,27,30,33,35}$ Self-Rating Depression Scale (SDS) in 4 studies, ${ }^{11-13,31}$ Symptom Checklist (SCL)-90 in 3 studies, ${ }^{22,23,29}$ and the Sphere score in 1 study. ${ }^{24}$ The anxiety score was assessed using the HADS in 15 studies, ${ }^{10,15-17,21,25,28,32,34-40}$ State and Trait Anxiety Inventory (STAI) in 5 studies, ${ }^{11,13,26,27,33}$ Self Rating Anxiety Scale (SAS) in 2 studies, ${ }^{12,31}$ SCL-90 score in 3 studies, ${ }^{22,29}$ Sphere score in 1 study, ${ }^{24}$ and the Beck Anxiety Inventory (BAI) in 1 study. ${ }^{30}$

\section{The Depression Level in Irritable Bowel Syndrome Patients}

Depression levels in IBS patients were higher than in healthy controls (Pooled SMD, 0.76; 95\% CI, 0.62-0.90; $P<0.001$; Fig. $2 \mathrm{~A})$. We used the random effect analysis because this result showed medium heterogeneity $\left(I^{2}=77.2 \%\right)$. Egger's test $(P=0.287)$ showed no small-study effects and the funnel plot showed no asymmetry, except for 8 outlier studies (Fig. 2B). ${ }^{11,13,22,25,28,29,34,36}$ However, 4 of 8 outlier studies were age-sex (hyphen) unmatched. ${ }^{13,28,29,34}$

\section{The Anxiety Level in Irritable Bowel Syndrome Patients}

Anxiety levels in IBS patients were also higher than in healthy controls (Pooled SMD, 0.84; 95\% CI, 0.67-1.01; $P<0.001$; Fig.
3A). We also used random effect analysis because this result showed high heterogeneity $\left(I^{2}=85.6 \%\right)$, Egger's test $(P=0.313)$ showed no small-study effects, and the funnel plot showed no asymmetry, except 9 outlier studies (Fig. 3B). ${ }^{10,11,13,22,25,28,29,35,36}$

\section{Meta-regression Analysis and Sub-group Analysis}

Meta-regression analysis was conducted due to medium and high heterogeneity. The result of meta-regression is shown in Table 2. In the meta-regression analysis, the heterogeneity of pooled $\mathrm{SMD}$ in the level of depression was due to the studied region (East Asia, USA, Europe, and others; $P=0.007$ ) and type of depression questionnaire used (HADS, BDI, SDS, and others; $P<0.001$ ). The heterogeneity of pooled SMD in the level of anxiety was also due to the studied region (East Asia, USA, Europe, and others; $P$ $=0.002$ ) and type of anxiety questionnaire used (HADS, STAI, SAS, and others; $P=0.007$ ). Pooled SMD according to subgroup analyses is also shown in Table 2. Wherein depression levels are assessed through a subgroup analysis, studies using the HADS (pooled SMD, 1.02; 95\% CI, 0.83-1.21; $I^{2}=66.3 \%$ ) and the BDI (pooled SMD, 0.71; 95\% CI, 0.52-0.90; $I^{2}=0 \%$ ) showed higher SMD than other questionnaires. Furthermore, studies conducted in East Asia showed the lowest pooled SMD (pooled 
Table 1. Characteristics of Studies Included in the Meta-analysis

\begin{tabular}{|c|c|c|c|c|c|c|c|c|c|}
\hline \multirow{2}{*}{ Study author } & \multirow{2}{*}{ Year } & \multirow{2}{*}{ Country } & \multirow{2}{*}{$\begin{array}{l}\text { Criteria } \\
\text { for IBS }\end{array}$} & \multirow{2}{*}{$\begin{array}{c}\text { Newcastle- } \\
\text { Ottawa } \\
\text { Scale }\end{array}$} & \multicolumn{2}{|c|}{ Questionnaire score } & \multicolumn{2}{|c|}{ Sample size } & \multirow{2}{*}{$\begin{array}{c}\text { Female } \\
\text { proportion in } \\
\text { IBS group }(\%)\end{array}$} \\
\hline & & & & & Depression & Anxiety & IBS & Control & \\
\hline Boyce et $\mathrm{al}^{24}$ & 2000 & Australia & Rome II & 6 & Sphere & Sphere & 201 & 2512 & 68.7 \\
\hline Pinto et $\mathrm{al}^{25}$ & 2000 & India & Manning & 6 & HADS & HADS & 30 & 30 & 33.3 \\
\hline Su et $\mathrm{al}^{26}$ & 2000 & Taiwan & Rome I & 5 & BDI & STAI & 69 & 52 & 49.0 \\
\hline Patacchioli et $\mathrm{al}^{27}$ & 2001 & Italy & Manning & 6 & BDI & STAI & 55 & 28 & 61.0 \\
\hline Locke et $\mathrm{al}^{23}$ & 2004 & USA & BDQ & 5 & SCL-90 & SCL-90 & 69 & 119 & 73 \\
\hline Liebregts et $\mathrm{al}^{28}$ & 2007 & Australia & Rome II & 5 & HADS & HADS & 55 & 36 & 60.0 \\
\hline Van der veek et $\mathrm{al}^{29}$ & 2008 & Netherlands & Rome II & 6 & SCL-90 & SCL-90 & 101 & 40 & 73.0 \\
\hline Choung et $\mathrm{al}^{22}$ & 2009 & USA & BDQ & 8 & SCL-90 & SCL-90 & 106 & 355 & 49.1 \\
\hline Savas et $\mathrm{al}^{30}$ & 2009 & USA & BDQ & 6 & BDI-II & $\mathrm{BAI}$ & 93 & 140 & 100 \\
\hline Shen et $\mathrm{al}^{31}$ & 2009 & China & Rome II & 6 & SDS & SAS & 77 & 414 & 54.5 \\
\hline Elsenbruch et $\mathrm{al}^{32}$ & 2010 & Germany & Rome III & 5 & HADS & HADS & 15 & 12 & 100 \\
\hline Heymen et $\mathrm{al}^{33}$ & 2010 & USA & Rome II & 5 & BDI & STAI & 27 & 21 & 100 \\
\hline Jerndal et $\mathrm{al}^{34}$ & 2010 & Sweden & Rome II & 6 & HADS & HADS & 306 & 60 & 76.7 \\
\hline Seminowicz et $\mathrm{al}^{17}$ & 2010 & USA & Rome II & 5 & HADS & HADS & 55 & 48 & 100 \\
\hline Tosic-Golubovic et $\mathrm{al}^{36}$ & 2010 & Serbia & Rome II & 5 & HADS & HADS & 30 & 30 & 50 \\
\hline Piche et $\mathrm{al}^{35}$ & 2010 & France & Rome III & 5 & BDI & HADS & 40 & 20 & 30 \\
\hline Cho et $\mathrm{al}^{37}$ & 2011 & Korea & Rome II & 6 & HADS & HADS & 124 & 91 & 49.2 \\
\hline Berman et $\mathrm{al}^{21}$ & 2012 & USA & Rome II & 6 & HADS & HADS & 11 & 11 & 54.5 \\
\hline Goncalves de Medeiros et $\mathrm{al}^{38}$ & 2012 & Brazil & Rome II & 5 & HADS & HADS & 21 & 8 & 76.2 \\
\hline Hartono et $\mathrm{al}^{39}$ & 2012 & Malaysia & Rome III & 5 & HADS & HADS & 62 & 62 & 55 \\
\hline Lee et $\mathrm{al}^{40}$ & 2012 & Taiwan & Rome III & 6 & HADS & HADS & 17 & 17 & 64.7 \\
\hline Orand et $\mathrm{al}^{10}$ & 2015 & USA & Rome III & 5 & HADS & HADS & 277 & 382 & 75.2 \\
\hline Komuro et $\mathrm{al}^{11}$ & 2016 & Japan & Rome III & 5 & SDS & STAI & 142 & 142 & 56.3 \\
\hline Qi et $\mathrm{al}^{12}$ & 2016 & China & Rome III & 7 & SDS & SAS & 32 & 31 & 19.4 \\
\hline Sasaki et $\mathrm{al}^{13}$ & 2016 & Japan & Rome III & 5 & SDS & STAI & 111 & 142 & 57.7 \\
\hline Thijssen et $\mathrm{al}^{15}$ & 2016 & Netherlands & Rome III & 6 & HADS & HADS & 154 & 137 & 70.0 \\
\hline Wong et $\mathrm{al}^{16}$ & 2016 & Singapore & Rome III & 5 & HADS & HADS & 13 & 11 & 53.8 \\
\hline
\end{tabular}

IBS, irritable bowel syndrome; BAI, Beck Anxiety Inventory; BDI, Beck Depression Inventory; HADS, Hospitalization Anxiety and Depression Scale; SAS, SelfRating Anxiety Scale; SCL-90, Symptom Checklist-90; SDS, Self-Rating Depression Scale; STAI, State and Trait Anxiety Inventory.

SMD, 0.50; 95\% CI, 0.32-0.69; $\left.I^{2}=56.3 \%\right)$. Wherein anxiety levels were assessed through a subgroup analysis, studies using the STAI and the SAS showed low heterogeneity $\left(I^{2}=48.5 \%\right.$ and $I^{2}$ $=0.0 \%$, respectively); those conducted in East Asia also showed the lowest pooled SMD (pooled SMD, 0.51; 95\% CI, 0.29-0.73; $\left.I^{2}=68.5 \%\right)$.

\section{Depression and Anxiety in Irritable Bowel Syndrome-subtype}

Depression and anxiety levels in IBS-subtype patients were also analyzed. A total of six studies were included. ${ }^{15,17,18,37,38,65}$ In those studies, healthy-control groups were used for multiple comparisons with the three IBS-subtypes; we divided the healthy-control group into three groups for meta-analysis of three IBS-subtypes alongside healthy controls. Depression levels were significantly higher in IBS$\mathrm{C}\left(\mathrm{SMD}=0.83 ; 95 \% \mathrm{CI}, 0.61-1.06 ; I^{2}=0 \% ; P<0.001\right)$, IBS$\mathrm{D}\left(\mathrm{SMD}=0.73 ; 95 \% \mathrm{CI}, 0.51-0.96 ; I^{2}=0 \% ; P<0.001\right)$, and IBS with mixed bowel habits $(\mathrm{SMD}=0.62 ; 95 \% \mathrm{CI}, 0.39-0.84$; $\left.I^{2}=0 \% ; P<0.001\right)$ (Fig. 4A). Egger's test $(P=0.323)$ showed no small-study effects and the funnel plot showed symmetry (Fig. 4B). Anxiety levels were also significantly higher in IBS-C (SMD $=0.81 ; 95 \%$ CI, 0.59-1.04; $\left.I^{2}=0 \% ; P<0.001\right)$, IBS-D (SMD $\left.=0.65 ; 95 \% \mathrm{CI}, 0.43-0.87 ; I^{2}=0 \% ; P<0.001\right)$, and IBS with mixed bowel habits (SMD $=0.75 ; 95 \% \mathrm{CI}, 0.52-0.97 ; I^{2}=0 \%$; $P<0.001)$ (Fig. 5A). Egger's test $(P=0.830)$ showed no smallstudy effects and the funnel plot showed symmetry (Fig. 5B). 
A

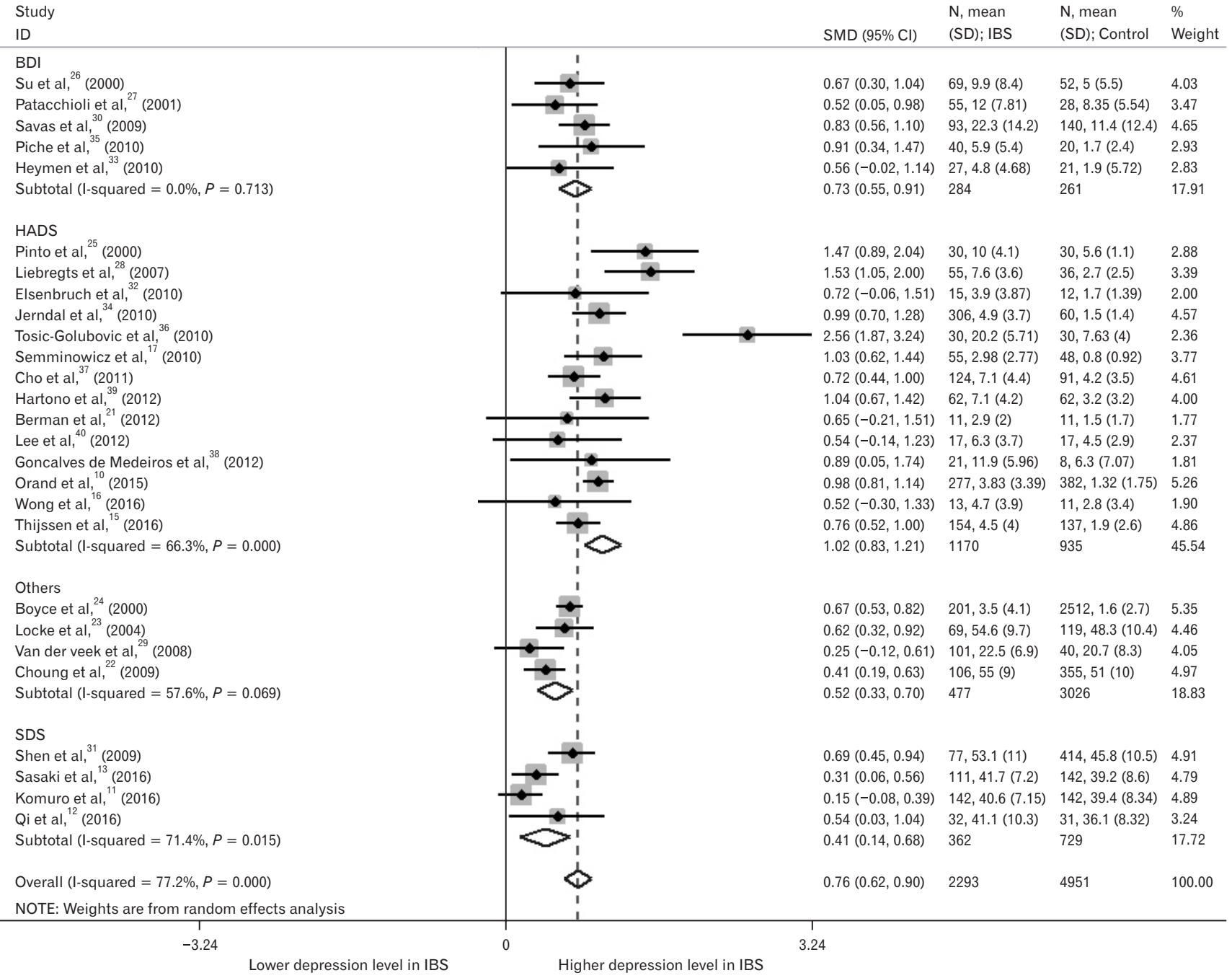

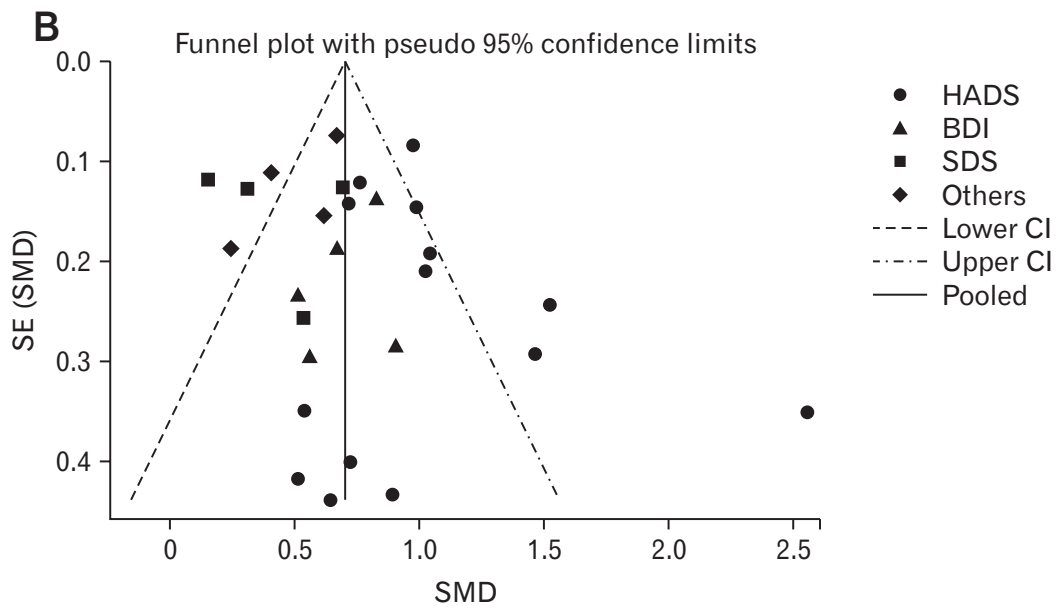

Figure 2. Forest plot and funnel plot of depression levels in irritable bowel syndrome (IBS) patients. (A) Forest plot of depression levels in IBS patients, divided by the type of questionnaire assessing depression levels. (B) Funnel plot of depression levels in IBS patients, divided by the type of questionnaire assessing depression levels. SMD, standard mean difference; BDI, Beck Depression Inventory; HADS, Hospitalization Anxiety and Depression Scale; SDS, Self-Rating Depression Scale; Others included sphere and Symptom Checklist-90; SE, standard error. 
A

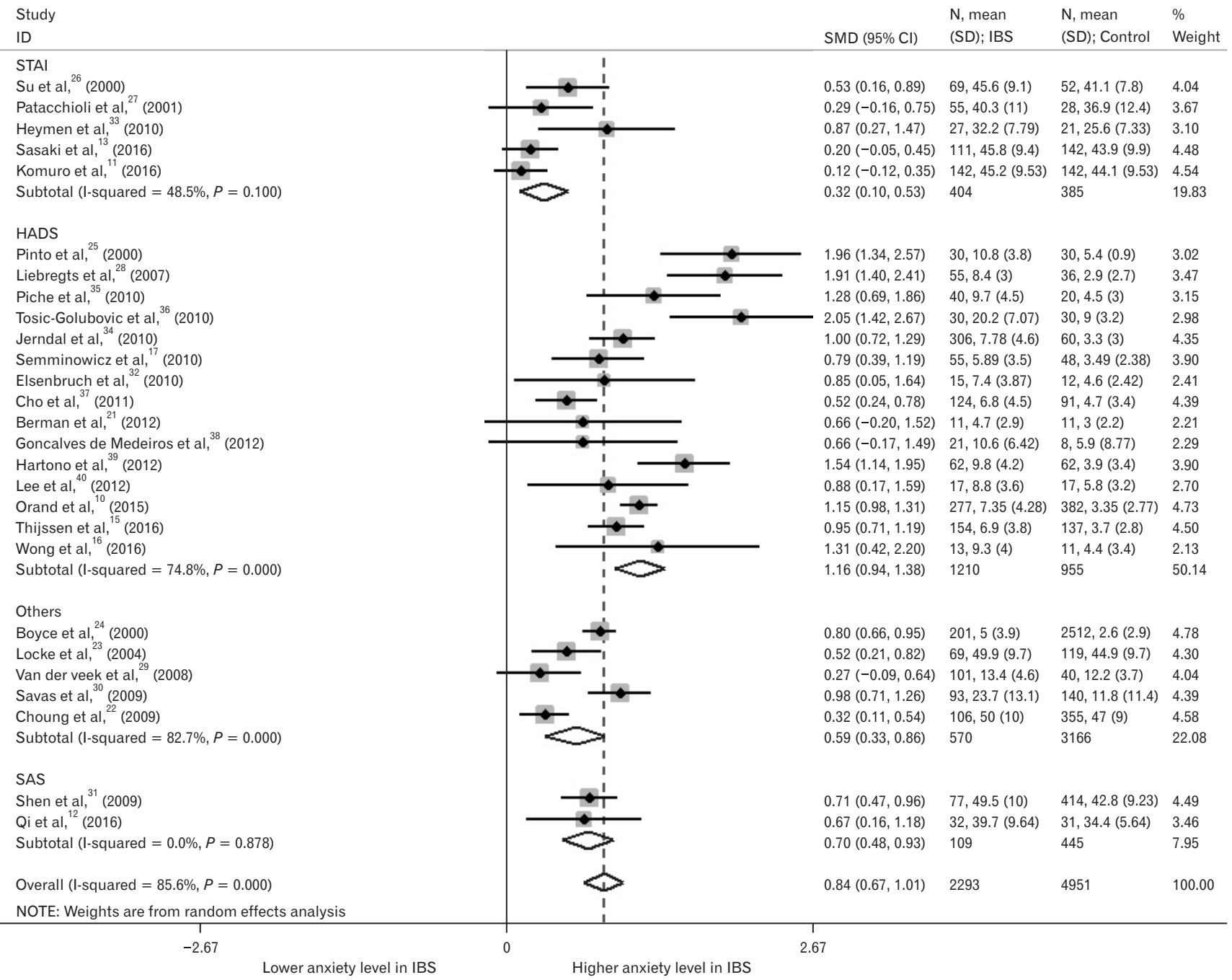

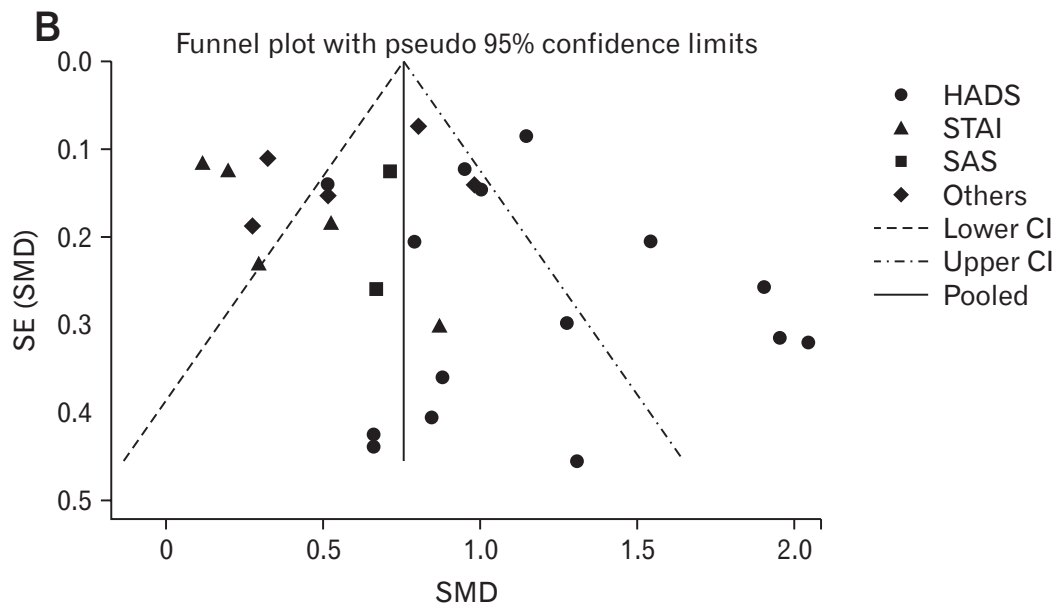

Figure 3. Forest plot and funnel plot of anxiety levels in irritable bowel syndrome (IBS) patients. (A) Forest plot of anxiety level in IBS patients, divided by the type of questionnaire assessing anxiety levels. (B) Funnel plot of anxiety levels in IBS patients, divided by the type of questionnaire assessing anxiety levels. SMD, standard mean difference; STAI, State and Trait Anxiety Inventory; HADS, Hospitalization Anxiety and Depression Scale; SAS, Self-Rating Anxiety Scale; Others included sphere, Symptom Checklist-90 and BAI; SE, standard error. 
Table 2. The Result of Meta-regression Analysis and the Pooled Standard Mean Difference for Depression and Anxiety Levels According to Subgroup Analysis

\begin{tabular}{|c|c|c|c|c|c|c|c|}
\hline & \multirow{2}{*}{ Subgroup } & \multirow{2}{*}{$\begin{array}{l}\text { No. of } \\
\text { studies }\end{array}$} & \multirow{2}{*}{ SMD (95\% CI) } & \multirow{2}{*}{$P$-value } & \multicolumn{2}{|c|}{ Heterogeneity } & \multirow{2}{*}{$\begin{array}{c}\text { Meta-regression } \\
\text { P-value }\end{array}$} \\
\hline & & & & & $I^{2}(\%)$ & $P_{\mathrm{h}}^{\mathrm{a}}$ & \\
\hline \multirow[t]{15}{*}{ Depression } & Overall & 27 & $0.76(0.62-0.90)$ & $<0.001$ & 77.2 & $<0.001$ & \\
\hline & Studied region & & & & & & 0.007 \\
\hline & East Asia & 8 & $0.50(0.32-0.69)$ & $<0.001$ & 56.3 & 0.025 & \\
\hline & USA & 7 & $0.74(0.52-0.96)$ & $<0.003$ & 77.2 & 0.003 & \\
\hline & Europe & 7 & $0.91(0.52-1.30)$ & $<0.001$ & 83.9 & $<0.001$ & \\
\hline & Others ${ }^{\mathrm{b}}$ & 5 & $1.10(0.70-1.49)$ & $<0.001$ & 78.6 & 0.001 & \\
\hline & IBS criteria & & & & & & 0.197 \\
\hline & Rome II & 11 & $0.91(0.60-1.15)$ & $<0.001$ & 80.0 & $<0.001$ & \\
\hline & Rome III & 10 & $0.65(0.40-0.90)$ & $<0.001$ & 80.4 & $<0.001$ & \\
\hline & Others ${ }^{c}$ & 6 & $0.70(0.46-0.93)$ & $<0.001$ & 65.4 & 0.013 & \\
\hline & The type of depression questionnaire & & & & & & $<0.001$ \\
\hline & HADS & 14 & $1.02(0.83-1.21)$ & $<0.001$ & 66.3 & $<0.001$ & \\
\hline & BDI & 5 & $0.73(0.55-0.91)$ & $<0.001$ & 0.0 & 0.713 & \\
\hline & SDS & 4 & $0.41(0.14-0.68)$ & $<0.001$ & 71.4 & 0.015 & \\
\hline & Others & 4 & $0.52(0.33-0.70)$ & $<0.001$ & 57.6 & 0.069 & \\
\hline \multirow[t]{15}{*}{ Anxiety } & Overall & 27 & $0.84(0.67-1.01)$ & $<0.001$ & 85.6 & $<0.001$ & \\
\hline & Studied region & & & & & & 0.002 \\
\hline & East Asia & 8 & $0.51(0.29-0.73)$ & $<0.001$ & 68.5 & 0.002 & \\
\hline & USA & 7 & $0.76(0.45-1.07)$ & $<0.001$ & 85.0 & $<0.001$ & \\
\hline & Europe & 7 & $0.92(0.55-1.29)$ & $<0.001$ & 74.8 & $<0.001$ & \\
\hline & Others $^{\mathrm{b}}$ & 5 & $1.38(0.81-1.95)$ & $<0.001$ & 88.9 & $<0.001$ & \\
\hline & IBS criteria & & & & & & 0.997 \\
\hline & Rome II & 11 & $0.90(0.66-1.14)$ & $<0.001$ & 79.2 & $<0.001$ & \\
\hline & Rome III & 10 & $0.86(0.51-1.21)$ & $<0.001$ & 90.1 & $<0.001$ & \\
\hline & Others ${ }^{c}$ & 6 & $0.72(0.36-1.07)$ & $<0.001$ & 85.3 & $<0.001$ & \\
\hline & The type of anxiety questionnaire & & & & & & 0.007 \\
\hline & HADS & 15 & $1.16(0.94-1.38)$ & $<0.001$ & 74.8 & $<0.001$ & \\
\hline & STAI & 5 & $0.32(0.10-0.53)$ & 0.004 & 48.5 & 0.100 & \\
\hline & SAS & 2 & $0.70(0.48-0.93)$ & $<0.001$ & 0.0 & 0.878 & \\
\hline & Others & 5 & $0.59(0.33-0.86)$ & $<0.001$ & 82.7 & $<0.001$ & \\
\hline
\end{tabular}

${ }^{a} P$-value of heterogeneity analysis.

${ }^{\mathrm{b}}$ Two studies in Australia, 1 in Brazil, 1 in India, and 1 in Malaysia.

'Three studies using the Bowel Disease Questionnaire, 2 studies using Manning's criteria and 1 study using Rome I criteria.

SMD, standard mean difference; IBS, irritable bowel syndrome; BDI, Beck Depression Inventory; HADS, Hospitalization Anxiety and Depression Scale; SAS, Self-Rating Anxiety Scale; SDS, Self-Rating Depression Scale; STAI, State and Trait Anxiety Inventory.

\section{Discussion}

We found that depression and anxiety levels in IBS patients were significantly higher than in healthy controls, even in the subgroup analyses with IBS-subtypes. Our studies also showed significant heterogeneity of these findings (depression, $I^{2}=77.2 \%$; anxiety, $I^{2}=85.6 \%$ ), however, we found the study region and type of questionnaire used to assess depression or anxiety levels to be the main causes of heterogeneity, using meta-regression analysis.

We studied the level of depression and anxiety, not prevalence, because of the continuing dispute on the threshold of questionnaires in patients with a medical illness. Patients with medical symptoms may record a high score that inappropriately suggests depression. Only the HADS has been reported to be suitable for measuring depression in patients with a known medical illness, because it was specially designed to overcome the said difficulty by omitting somatic symptoms. ${ }^{66}$ The other questionnaires might overestimate the 
A

Study

ID

IBS-C

Camillari et al, ${ }^{65}$ (2008)

Semminowicz et al, ${ }^{17}$ (2010)

Cho et al, ${ }^{37}$ (2011)

Goncalves et al, ${ }^{38}$ (2012)

Jones et al, ${ }^{18}$ (2014)

Thijssen et al, ${ }^{15}$ (2016)

Subtotal (I-squared $=0.0 \%, P=0.461$ )

IBS-D

Camillari et al, ${ }^{65}$ (2008)

Semminowicz et al, ${ }^{17}$ (2010)

Cho et al, ${ }^{37}$ (2011)

Goncalves et al, ${ }^{38}$ (2012)

Jones et al, ${ }^{18}$ (2014)

Thijssen et al, ${ }^{15}$ (2016)

Subtotal (I-squared $=0.0 \%, P=0.881$ )

IBS-M

Camillari et al, ${ }^{65}$ (2008)

Semminowicz et al, ${ }^{17}$ (2010)

Cho et al, ${ }^{37}$ (2011)

Goncalves et al, ${ }^{38}$ (2012)

Jones et al, ${ }^{18}$ (2014)

Thijssen et al, ${ }^{15}$ (2016)

Subtotal (I-squared $=0.0 \%, P=0.790)$

Overall (I-squared $=0.0 \%, P=0.874$ )

NOTE: Weights are from random effects analysis

\begin{tabular}{|c|c|c|}
\hline NOTE: Weights are from random effects analysis & & \\
\hline 1 & & $T$ \\
\hline-2.83 & 0 & 2.83 \\
\hline
\end{tabular}

(SD); IBS

$\mathrm{N}$, mean

$\%$

$\operatorname{SMD}(95 \% \mathrm{Cl})$

(S)

$0.57(-0.04,1.18) \quad 44,1.6(2.1)$

$1.34(0.58,2.10) \quad 17,3.27(2.4)$

$14,0.5(1.28) \quad 4.43$

$0.99(0.53,1.45) \quad 63,8.8(5.1) \quad 30,4.2(3.5) \quad 7.87$

$0.76(-0.68,2.20) \quad 6,11(5.82) \quad 3,6.3(7.07) \quad 0.79$

$0.53(0.05,1.00) \quad 57,3.3(3.98) \quad 25,1.47(1.85) \quad 7.24$

$0.91(0.49,1.33) \quad 52,4.9(3.8) \quad 46,1.9(2.6) \quad 9.48$

$0.83(0.61,1.06) \quad 239 \quad 134 \quad 32.66$

$0.68(0.07,1.30) \quad 44,1.4(1.33) \quad 14,0.5(1.28) \quad 4.37$

$1.07(0.33,1.80) \quad 17,3.06(2.81) \quad 16,0.8(0.922) \quad 3.07$

$0.67(0.23,1.12) \quad 63,6.6(3.6) \quad 30,4.2(3.5) \quad 8.31$

$0.58(-0.84,2.00) \quad 6,10.2(6.61) \quad 3,6.3(7.07) \quad 0.82$

$0.56(0.08,1.04) \quad 57,3.07(3.2) \quad 25,1.47(1.85) \quad 7.22$

$0.85(0.44,1.27) \quad 52,4.6(3.6) \quad 46,1.9(2.6) \quad 9.60$

$\begin{array}{llll}0.73(0.51,1.96) & 239 & 134 & 33.38\end{array}$

$0.71(0.09,1.32) \quad 44,1.9(2.15) \quad 14,0.5(1.28) \quad 4.35$

$0.84(0.13,1.56) \quad 17,2.21(2.15) \quad 16,0.8(0.922) \quad 3.23$

$0.51(0.06,0.95) \quad 63,6.4(4.7) \quad 30,4.2(3.5) \quad 8.48$

$1.29(-0.25,2.83) \quad 6,13.9(5.37) \quad 3,6.3(7.07) \quad 0.69$

$0.40(-0.07,0.88) \quad 57,2.41(2.52) \quad 25,1.47(1.85) \quad 7.33$

$0.71(0.30,1.12) \quad 52,4.4(4.2) \quad 46,1.9(2.6) \quad 9.86$

$\begin{array}{llll}0.62(0.39,0.84) & 239 & 134 & 33.96\end{array}$

$\begin{array}{llll}0.73(0.60,0.85) & 717 & 402 & 100.00\end{array}$

B Funnel plot with pseudo $95 \%$ confidence limits

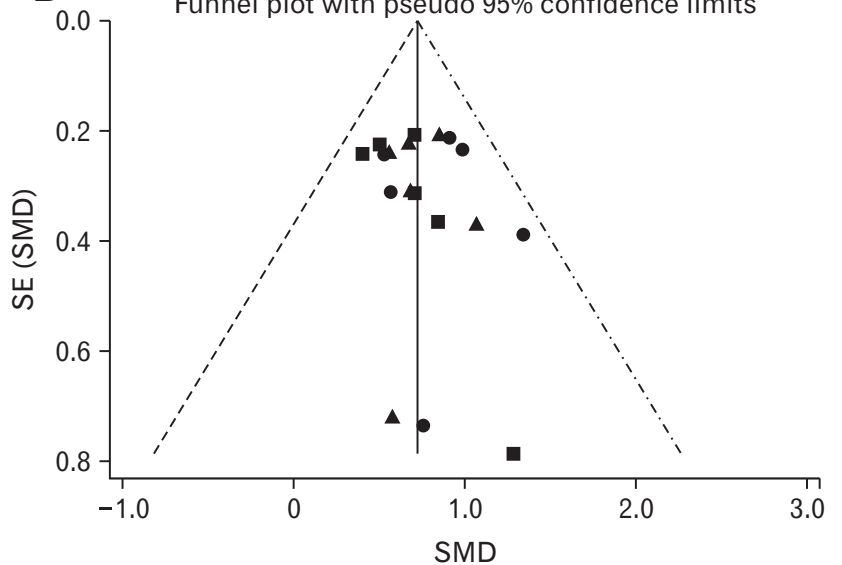

- IBS-C

- IBS-D

- IBS-M

-.-- Lower Cl

-.-.- Upper C

- Pooled

Figure 4. Forest plot and funnel plot of depression levels in subtypes of irritable bowel syndrome (IBS). (A) Forest plot of depression levels in IBS-subtype patients (B) Funnel plot of depression levels in IBS-subtype patients. SMD, standard mean difference; IBS-C, IBS with predominant constipation; IBS-D, IBS with predominant diarrhea; IBS-M, IBS with mixed bowel habits; SE, standard error. 
A

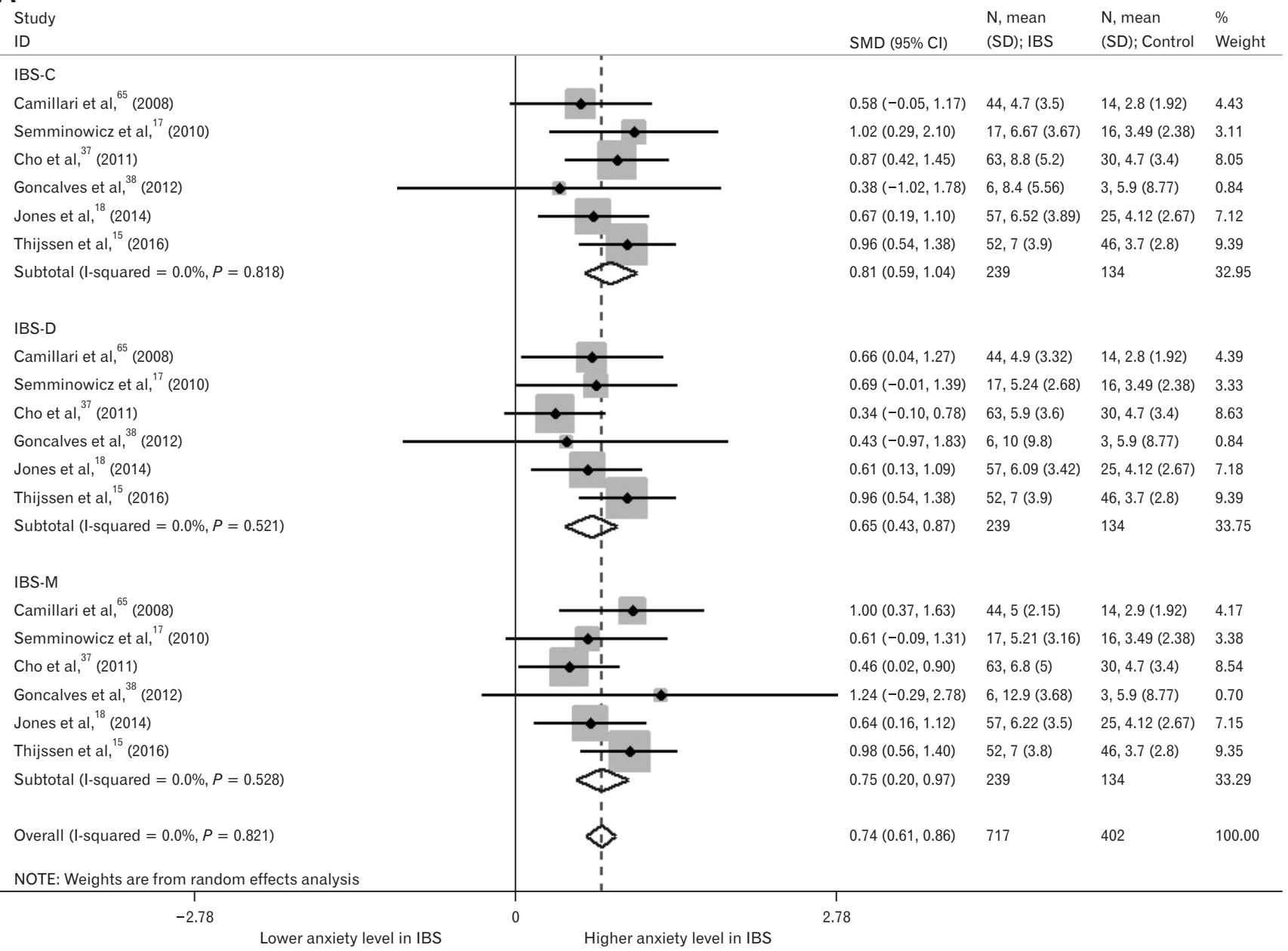

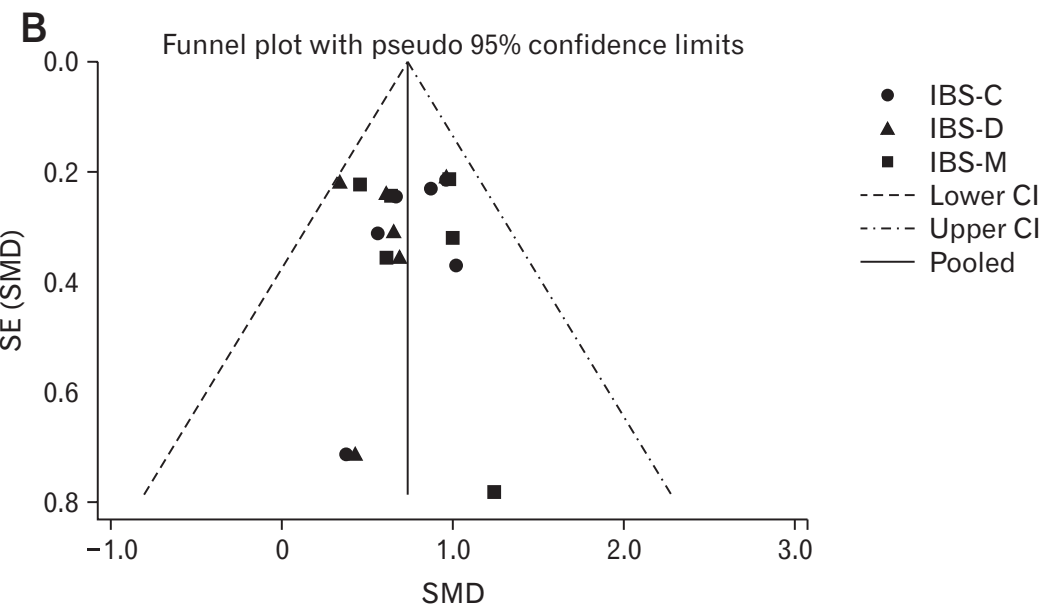

Figure 5. Forest plot and funnel plot of depression levels in subtypes of irritable bowel syndrome (IBS). (A) Forest plot of anxiety levels in IBSsubtype patients. (B) Funnel plot of anxiety levels in IBS-subtype patients. SMD, standard mean difference; IBS-C, IBS with predominant constipation; IBS-D, IBS with predominant diarrhea; IBS-M, IBS with mixed bowel habits; SE, standard error. 
symptoms of depression because they do not rule out somatic questions. However, the HADS is a symptom-screening questionnaire, and not a diagnostic measure for depression. Furthermore, pooled SMD of the HADS was higher than other questionnaires in metaregression analysis of our study. There is still no consensus on this issue.

The studied regions contributed to the significant heterogeneity found in this meta-analysis. The prevalence of IBS, anxiety, and depression varies according to the cultural region. This diversity in prevalence may be due to actual differences in prevalence, discrepancies in measurement, due to translation, and limited validation of questionnaires in question in each cultural region, or all of them. ${ }^{67}$ Cross-cultural factors are considered important, not only in the diagnosis of anxiety and depression, but also in IBS, as symptoms are also vague and there are no objective findings to confirm the diagnoses. Furthermore, the translation of words used to describe symptoms into another language introduces complexities, because there are sometimes no exact equivalents. Therefore, the translated questionnaires used to diagnose IBS and the questionnaires used to assess anxiety and depression levels would contribute towards heterogeneity in this meta-analysis.

The newly revised Rome IV highlights this importance of cross-cultural competence in clinical medicine and research. ${ }^{68}$ The English version of diagnostic questionnaire for IBS in the Rome IV criteria has been translated into other languages and is being validated; this should make it possible to carry out cross-cultural and global epidemiologic studies. ${ }^{69}$ However, Rome IV criteria were not used in enrolled studies. Several types of diagnostic criteria for IBS were used in studies selected in this meta-analysis, but the types of IBS criteria did not significantly influence heterogeneity.

There are also cultural differences in the prevalence and diagnosis of anxiety and depression in different regions. Low prevalence rates of common mental disorders such as anxiety and depression in East Asia have been reported in a recent meta-analysis. ${ }^{70}$ In this meta-analysis, studies conducted in East Asia also showed lower pooled SMD in both depression and anxiety levels. The results of the WHO World Health Survey depression study also showed East Asia to have the lowest prevalence estimates. ${ }^{71}$ Reasons for the low prevalence of depression and anxiety in East Asia are still unknown, but both protective cultural factors and underestimation due to cultural differences could be possible explanations. ${ }^{70}$ Therefore, it is important to understand culture-specific ways to express mental symptoms. ${ }^{72}$ Consensus or guidelines must be proposed for the estimation of depression and anxiety levels in IBS patients from different cultural backgrounds, so cultural heterogeneity can be overcome.

In general, higher prevalence among women was not limited to IBS, but pertained to anxiety and depression as well. ${ }^{73}$ Therefore, women were considered to have more impaired quality of life with IBS, due to the correlation between somatic symptoms and a gender-related increase in anxiety and depression prevalence. ${ }^{73}$ To know the gender effect on psychological factors among IBS patients, we tried to get the gender specific score of anxiety and depression. However, it is not possible to get the gender specific data in this meta-analysis. As an alternative, we studied the heterogeneity of the results according to the proportion of female patients. Interestingly, the proportion of female patients did not affect heterogeneity in this meta-analysis. In the meta-analysis, most studies, except $3,{ }^{12,25,35}$ enrolled more than $49 \%$ of female patients; so, the proportion of female patients did not differ across studies. However, this finding means only that the proportion of female patients did not affect pooled SMD among the studies. The gender effect on psychological factors in IBS patients is still unknown because the data of either depression or anxiety levels between male and female patients in enrolled studies could not be acquired. A direct comparison between male and female patients was not possible in this study. The gender effect in IBS with psychological distress should be evaluated in prospective studies.

In the meta-analysis on IBS-subtype, both depression and anxiety levels were higher in all IBS-subtypes. In our study, IBS-C patients showed the highest $\mathrm{SMD}$ for depression $(\mathrm{SMD}=0.83$; 95\% CI, 0.61-1.06) and anxiety (SMD $=0.81 ; 95 \%$ CI, 0.591.04). One possible explanation for this finding is a change in the intestinal serotonin (5-hydroxytryptamine, 5-HT) system. Excess 5-HT could contribute to diarrhea through the 5-HT receptor; moreover, increased postprandial release of 5-HT in patients with IBS-D has been reported. ${ }^{74}$ On the other hand, the IBS-C subtype may be considered an imbalance of 5-HT secretion, and high levels of depression and anxiety in IBS-C subtype may be associated with low responsiveness of 5-HT in both the central and peripheral regions. ${ }^{75}$ However, all subtypes showed significantly higher depression and anxiety levels in our meta-analysis.

This review has some limitations. First, we included Englishlanguage studies only; so some articles may have been missed despite extensive database searches. Second, high heterogeneity may be attributable to the assessment methods and regions under study in the primary studies. This makes it difficult to draw consistent conclusions about anxiety and depression levels among IBS patients. There is a need for consensus about the evaluation methods of depression and anxiety in IBS patients. 
In conclusion, the present meta-analysis showed that depression and anxiety levels were higher in IBS patients than in healthy controls, regardless of IBS-subtype. However, the gender effect on psychological factors among IBS patients could not be determined. Further prospective studies are needed to evaluate and compare the mechanisms of those relationships.

\section{Supplementary Material}

Note: To access the supplementary table mentioned in this article, visit the online version of Journal of Neurogastroenterology and Motility at http://www.jnmjournal.org/, and at https://doi. org/10.5056/jnm16220.

Acknowledgements: The Seoul National University Hospital Medical Research Collaborating Center (SNUH MRCC) contributed to the review of statistical analysis.

Financial support: This study was supported by a grant from the Korean Society of Neurogastroenterology and Motility for 2016.

\section{Conflicts of interest: None.}

Author contributions: Changhyun Lee and Eunyoung Do: data collection, analysis and interpretation, and drafting the manuscript; Seung-ho Jang: data interpretation and draft revising; Ji Min Choi, Ju Yup Lee, Han-Seung Ryu, Jung Hwan Oh and Jung Ho Park: critical revision and final approval; and Yong Sung Kim: study concept, design of the work, critical review, and final approval.

\section{References}

1. Drossman DA. Functional gastrointestinal disorders: history, pathophysiology, clinical features and Rome IV. Gastroenterology 2016;150:12621279, e2.

2. Lovell RM, Ford AC. Global prevalence of and risk factors for irritable bowel syndrome: a meta-analysis. Clin Gastroenterol Hepatol 2012;10:712-721.e4.

3. Mearin F, Mearin F, Chang L, et al. Bowel disorders. Gastroenterology 2016;150:1393-1407, e1395.

4. Van Oudenhove L, Crowell MD, Drossman DA, et al. Biopsychosocial aspects of functional gastrointestinal disorders: how central and environmental processes contribute to the development and expression of functional gastrointestinal disorders. Gastroenterology 2016;150:1355-1367, e2.
5. Lee SP, Sung IK, Kim JH, Lee SY, Park HS, Shim CS. The effect of emotional stress and depression on the prevalence of digestive diseases. J Neurogastroenterol Motil 2015;21:273-282.

6. Houghton LA, Calvert EL, Jackson NA, Cooper P, Whorwell PJ. Visceral sensation and emotion: a study using hypnosis. Gut 2002;51:701704.

7. Wilder-Smith $\mathrm{CH}$. The balancing act: endogenous modulation of pain in functional gastrointestinal disorders. Gut 2011;60:1589-1599.

8. Bennett EJ, Tennant CC, Piesse C, Badcock CA, Kellow JE. Level of chronic life stress predicts clinical outcome in irritable bowel syndrome. Gut 1998;43:256-261.

9. Coss-Adame E, Rao SS. Brain and gut interactions in irritable bowel syndrome: new paradigms and new understandings. Curr Gastroenterol Rep 2014;16:379.

10. Orand A, Gupta A, Shih W, et al. Catecholaminergic gene polymorphisms are associated with GI symptoms and morphological brain changes in irritable bowel syndrome. PLoS One 2015;10:e0135910.

11. Komuro H, Sato N, Sasaki A, et al. Corticotropin-releasing hormone receptor 2 gene variants in irritable bowel syndrome. PLoS One 2016;11:e0147817.

12. Qi R, Liu C, Ke J, et al. Abnormal amygdala resting-state functional connectivity in irritable bowel syndrome. AJNR Am J Neuroradiol 2016;37:1139-1145

13. Sasaki A, Sato N, Suzuki N, et al. Associations between single-nucleotide polymorphisms in corticotropin-releasing hormone-related genes and irritable bowel syndrome. PLoS One 2016;11:e0149322.

14. Schneck AS, Anty R, Tran A, et al. Increased prevalence of irritable bowel syndrome in a cohort of french morbidly obese patients candidate for bariatric surgery. Obes Surg 2016;26:1525-1530.

15. Thijssen AY, Mujagic Z, Jonkers DM, et al. Alterations in serotonin metabolism in the irritable bowel syndrome. Aliment Pharmacol Ther 2016;43:272-282.

16. Wong RK, Van Oudenhove L, Li X, Cao Y, Ho KY, Wilder-Smith CH. Visceral pain perception in patients with irritable bowel syndrome and healthy volunteers is affected by the MRI scanner environment. United European Gastroenterol J 2016;4:132-141.

17. Seminowicz DA, Labus JS, Bueller JA, et al. Regional gray matter density changes in brains of patients with irritable bowel syndrome. Gastroenterology 2010;139:48-57, e2.

18. Jones MP, Chey WD, Singh S, et al. A biomarker panel and psychological morbidity differentiates the irritable bowel syndrome from health and provides novel pathophysiological leads. Aliment Pharmacol Ther 2014;39:426-437.

19. Fond G, Loundou A, Hamdani N, et al. Anxiety and depression comorbidities in irritable bowel syndrome (IBS): a systematic review and metaanalysis. Eur Arch Psychiatry Clin Neurosci 2014;264:651-660.

20. Wells G, Shea B, O'Connell D, Peterson J, et al. The Newcastle-Ottawa Scale (NOS) for assessing the quality if nonrandomized studies in metaanalyses. Available from: URL: http://www.ohri.ca/programs/clinical epidemiology/oxford.asp (accessed 11 June 2017).

21. Berman S, Suyenobu B, Naliboff BD, et al. Evidence for alterations in central noradrenergic signaling in irritable bowel syndrome. Neuroimage 
2012;63:1854-1863.

22. Choung RS, Locke GR 3rd, Zinsmeister AR, Schleck CD, Talley NJ. Psychosocial distress and somatic symptoms in community subjects with irritable bowel syndrome: a psychological component is the rule. Am J Gastroenterol 2009;104:1772-1779.

23. Locke GR 3rd, Weaver AL, Melton LJ 3rd, Talley NJ. Psychosocial factors are linked to functional gastrointestinal disorders: a population based nested case-control study. Am J Gastroenterol 2004;99:350-357.

24. Boyce PM, Koloski NA, Talley NJ. Irritable bowel syndrome according to varying diagnostic criteria: are the new Rome II criteria unnecessarily restrictive for research and practice? Am J Gastroenterol 2000;95:31763183.

25. Pinto C, Lele MV, Joglekar AS, Panwar VS, Dhavale HS. Stressful lifeevents, anxiety, depression and coping in patients of irritable bowel syndrome. J Assoc Physicians India 2000;48:589-593.

26. Su YC, Wang WM, Wang SY, et al. The association between Helicobacter pylori infection and functional dyspepsia in patients with irritable bowel syndrome. Am J Gastroenterol 2000;95:1900-1905.

27. Patacchioli FR, Angelucci L, Dellerba G, Monnazzi P, Leri O. Actual stress, psychopathology and salivary cortisol levels in the irritable bowel syndrome (IBS). J Endocrinol Invest 2001;24:173-177.

28. Liebregts T, Adam B, Bredack C, et al. Immune activation in patients with irritable bowel syndrome. Gastroenterology 2007;132:913-920.

29. van der Veek PP, Van Rood YR, Masclee AA. Symptom severity but not psychopathology predicts visceral hypersensitivity in irritable bowel syndrome. Clin Gastroenterol Hepatol 2008;6:321-328.

30. Savas LS, White DL, Wieman M, et al. Irritable bowel syndrome and dyspepsia among women veterans: prevalence and association with psychological distress. Aliment Pharmacol Ther 2009;29:115-125.

31. Shen L, Kong H, Hou X. Prevalence of irritable bowel syndrome and its relationship with psychological stress status in Chinese university students. J Gastroenterol Hepatol 2009;24:1885-1890.

32. Elsenbruch S, Rosenberger C, Enck P, Forsting M, Schedlowski M, Gizewski ER. Affective disturbances modulate the neural processing of visceral pain stimuli in irritable bowel syndrome: an fMRI study. Gut 2010;59:489-495.

33. Heymen S, Maixner W, Whitehead WE, Klatzkin RR, Mechlin B, Light KC. Central processing of noxious somatic stimuli in patients with irritable bowel syndrome compared with healthy controls. Clin J Pain 2010;26:104-109.

34. Jerndal P, Ringström G, Agerforz P, et al. Gastrointestinal-specific anxiety: an important factor for severity of GI symptoms and quality of life in IBS. Neurogastroenterol Motil 2010;22:646-e179.

35. Piche T, Ducrotté P, Sabate JM, et al. Impact of functional bowel symptoms on quality of life and fatigue in quiescent Crohn disease and irritable bowel syndrome. Neurogastroenterol Motil 2010;22:626-e174.

36. Tosic-Golubovic S, Miljkovic S, Nagorni A, Lazarevic D, Nikolic G. Irritable bowel syndrome, anxiety, depression and personality characteristics. Psychiatr Danub 2010;22:418-424.

37. Cho HS, Park JM, Lim CH, et al. Anxiety, depression and quality of life in patients with irritable bowel syndrome. Gut Liver 2011;5:29-36.

38. Goncalves de Medeiros MT, de Oliveira RB, dos Santos AA, et al. The effects of sildenafil on rectal sensitivity and tone in patients with the irritable bowel syndrome. Aliment Pharmacol Ther 2012;35:577-586.

39. Hartono JL, Mahadeva S, Goh KL. Anxiety and depression in various functional gastrointestinal disorders: do differences exist? J Dig Dis 2012;13:252-257.

40. Lee HF, Hsieh JC, Lu CL, et al. Enhanced affect/cognition-related brain responses during visceral placebo analgesia in irritable bowel syndrome patients. Pain 2012;153:1301-1310.

41. Kabra N, Nadkarni A. Prevalence of depression and anxiety in irritable bowel syndrome: a clinic based study from India. Indian J Psychiatry 2013;55:77-80.

42. Park JH, Rhee PL, Kim HS, et al. Mucosal mast cell counts correlate with visceral hypersensitivity in patients with diarrhea predominant irritable bowel syndrome. J Gastroenterol Hepatol 2006;21:71-78.

43. Uz E, Türkay C, Aytac S, Bavbek N. Risk factors for irritable bowel syndrome in Turkish population: role of food allergy. J Clin Gastroenterol 2007;41:380-383.

44. Kohen R, Tracy JH, Haugen E, Cain KC, Jarrett ME, Heitkemper MM. Rare variants of the serotonin transporter are associated with psychiatric comorbidity in irritable bowel syndrome. Biol Res Nurs 2016;18:394-400.

45. Pinto-Sanchez MI, Ford AC, Avila CA, et al. Anxiety and depression increase in a stepwise manner in parallel with multiple FGIDs and symptom severity and frequency. Am J Gastroenterol 2015;110:1038-1048.

46. Mujagic Z, Ludidi S, Keszthelyi D, et al. Small intestinal permeability is increased in diarrhoea predominant IBS, while alterations in gastroduodenal permeability in all IBS subtypes are largely attributable to confounders. Aliment Pharmacol Ther 2014;40:288-297.

47. Sugaya N, Nomura S, Shimada H. Relationship between cognitive factors and anxiety in individuals with irritable bowel syndrome. Int J Behav Med 2012;19:308-315.

48. Hod K, Ringel-Kulka T, Martin CF, Maharshak N, Ringel Y. Highsensitive $\mathrm{C}$-reactive protein as a marker for inflammation in irritable bowel syndrome. J Clin Gastroenterol 2016;50:227-232.

49. Eriksson EM, Andrén KI, Eriksson HT, Kurlberg GK. Irritable bowel syndrome subtypes differ in body awareness, psychological symptoms and biochemical stress markers. World J Gastroenterol 2008;14:4889-4896.

50. Keszthelyi D, Troost FJ, Jonkers DM, Kruimel JW, Leue C, Masclee AA. Decreased levels of kynurenic acid in the intestinal mucosa of IBS patients: relation to serotonin and psychological state. J Psychosom Res 2013;74:501-504.

51. Grasberger H, Chang L, Shih W, et al. Identification of a functional TPH1 polymorphism associated with irritable bowel syndrome bowel habit subtypes. Am J Gastroenterol 2013;108:1766-1774.

52. Bouchoucha M, Hejnar M, Devroede G, Boubaya M, Bon C, Benamouzig R. Patients with irritable bowel syndrome and constipation are more depressed than patients with functional constipation. Dig Liver Dis 2014;46:213-218.

53. Kanuri N, Cassell B, Bruce SE, et al. The impact of abuse and mood on bowel symptoms and health-related quality of life in irritable bowel syndrome (IBS). Neurogastroenterol Motil 2016;28:1508-1517.

54. Padhy SK, Mishra S, Sarkar S, Bang LG, Panigrahi M. Comparison of 
psychiatric morbidity in patients with irritable bowel syndrome and nonulcer dyspepsia. Ind Psychiatry J 2016;25:29-34.

55. Roohafza H, Bidaki EZ, Hasanzadeh-Keshteli A, Daghaghzade H, Afshar H, Adibi P. Anxiety, depression and distress among irritable bowel syndrome and their subtypes: an epidemiological population based study. Adv Biomed Res 2016;5:183.

56. Gao J. Correlation between anxiety-depression status and cytokines in diarrhea-predominant irritable bowel syndrome. Exp Ther Med 2013;6:93-96.

57. Dunlop SP, Jenkins D, Spiller RC. Distinctive clinical, psychological, and histological features of postinfective irritable bowel syndrome. Am J Gastroenterol 2003;98:1578-1583.

58. Motzer SA, Hertig V, Jarrett M, Heitkemper MM. Sense of coherence and quality of life in women with and without irritable bowel syndrome. Nurs Res 2003;52:329-337.

59. Lee KJ, Kim YB, Kim JH, Kwon HC, Kim DK, Cho SW. The alteration of enterochromaffin cell, mast cell, and lamina propria T lymphocyte numbers in irritable bowel syndrome and its relationship with psychological factors. J Gastroenterol Hepatol 2008;23:1689-1694.

60. Hamaguchi T, Fukudo S, Kanazawa M, et al. Changes in salivary physiological stress markers induced by muscle stretching in patients with irritable bowel syndrome. Biopsychosoc Med 2008;2:20.

61. Akkuş S, Senol A, Ayvacioglu NB, Tunc E, Eren I, Isler M. Is female predominance in irritable bowel syndrome related to fibromyalgia? Rheumatol Int 2004;24:106-109.

62. Dancey CP, Attree EA, Stuart G, Wilson C, Sonnet A. Words fail me: the verbal IQ deficit in inflammatory bowel disease and irritable bowel syndrome. Inflamm Bowel Dis 2009;15:852-857.

63. Sato N, Suzuki N, Sasaki A, et al. Corticotropin-releasing hormone receptor 1 gene variants in irritable bowel syndrome. PLoS One 2012;7:e42450.

64. Qi R, Liu C, Ke J, et al. Intrinsic brain abnormalities in irritable bowel syndrome and effect of anxiety and depression. Brain Imaging Behav
2016;10:1127-1134

65. Camilleri M, McKinzie S, Busciglio I, et al. Prospective study of motor, sensory, psychologic, and autonomic functions in patients with irritable bowel syndrome. Clin Gastroenterol Hepatol 2008;6:772-781.

66. Cusin C, Yang H, Yeung A, Fava M. Rating scales for depression. Handbook of clinical rating scales and assessment in psychiatry and mental health. Springer 2009:7-35.

67. Lewis-Fernández R, Hinton DE, Laria AJ, et al. Culture and the anxiety disorder: recommendations for DSM-V. Depress Anxiety 2010;27:212229.

68. Fang X, Francisconi CF, Fukudo S, et al. Multicultural aspects in functional gastrointestinal disorders (FGIDs). Gastroenterology 2016:150;1344-1354.e2.

69. Palsson OS, Whitehead WE, van Tilburg MAL, et al. Development and validation of the Rome IV diagnostic questionnaire for adults. Gastroenterology 2016;150:1481-1491.

70. Steel Z, Marnane C, Iranpour C, et al. The global prevalence of common mental disorders: a systematic review and meta-analysis 1980-2013. Int J Epidemiol 2014;3:476-493.

71. Rai D, Zitko P, Jones K, Lynch J, Araya R. Country- and individuallevel socioeconomic determinants of depression: multilevel cross-national comparison. Br J Psychiatry 2013;202:195-203.

72. Steel Z, Silove D, Giao NM, et al. International and indigenous diagnoses of mental disorder among Vietnamese living in Vietnam and Australia. Br J Psychiatry 2009;194:326-333.

73. Houghton LA, Heitkemper M, Crowell M, et al. Age, gender and women's health and the patient. Gastroenterology 2016;150:1332-1343, e4.

74. Spiller R. Serotonin and GI clinical disorders. Neuropharmacology 2008;55:1072-1080

75. Muscatello MR, Bruno A, Pandolfo G, et al. Depression, anxiety and anger in subtypes of irritable bowel syndrome patients. J Clin Psychol Med Settings 2010;17:64-70. 